\title{
A Method of Optimizing Field Roll-Off and the Peak Field of Hybrid Planar Undulators
}

\author{
Melike Abliz, Isaac Vasserman
}

\begin{abstract}
Two new advancements have recently been made concerning hybrid planar undulator design. The first finding is how precisely the field roll-off in the x-direction can be controlled by altering the pole's xy chamfer dimension. The second finding is how effectively the peak field can be increased by use of a large pole $\mathrm{xx}$ chamfer. The detailed field calculations regarding these pole chamfers are reported in this technical note.

\section{Introduction}

Recently, a 17.2-mm-period hybrid undulator was designed as part of the upgrade project at the APS. Through modeling of the 17.2-mm-period hybrid undulator, the pole's $\mathrm{x}$ chamfers were investigated, and it was found that they can be optimized to control the field roll-off. This also affects the peak field of an undulator. The peak field of the 17.2-mm-period undulator was increased by about $1.5 \%$, compared to a conventional chamfer, when the pole's x chamfers were optimized with the same 10.4-mm gap. All other dimensions of the pole and magnet remained the same for the optimizing pole's $\mathrm{x}$ chamfers.
\end{abstract}

\section{Results}

A magnetic design of a quarter-period-long undulator with a 17.2-mm period was built with Opera as shown in Fig. 1. The peak field plot versus the pole's xx and xy chamfer dimensions is shown in the 3D plot of Fig. 2. Permanent magnet grade N42SH (NdFeB-type permanent magnet grade from Shin-Etsu) and vanadium permendur were used for the magnet and pole materials, respectively.

The peak field increases when the pole's xx chamfer dimension increases, while the peak field decreases when the pole's xy chamfer dimension increases. Therefore, the peak field is calculated only as a function of the pole's xx chamfer; the pole's xy chamfer is fixed at $0.2 \mathrm{~cm}$ in Fig. 3. The peak field increases with the pole's increasing $\mathrm{xx}$ chamfer up to $1.5 \mathrm{~cm}$, then it decreases rapidly.

Figure 4 shows the By field's roll-off dependence on the pole's $\mathrm{xx}$ chamfer. The roll-off field remains below a constant value about of $2 \times 10^{-3}$ as the pole's xx chamfer increases up to $1.2 \mathrm{~cm}$, then it increases rapidly. The results of Fig. 3 and Fig. 4 validate that the xx and xy chamfers provides maximum peak field and a low field roll-off would be $1.2 \mathrm{~cm}$ and $0.2 \mathrm{~cm}$ for the $17.2 \mathrm{~mm}$ period undulator. The required field roll-off is $1.9 \times 10^{-3}$ for the 17.2-mm-period undulator.

Figure 5 shows the calculated maximum demagnetization field, $\mathrm{H}_{\mathrm{z}}$, in the permanent magnet block with the pole's xx and xy chamfers of $1.2 \mathrm{~cm}$ and $0.2 \mathrm{~cm}$, respectively, at the same gap of $10.4 \mathrm{~mm}$ with a $17.2-\mathrm{mm}-$ period undulator. The demagnetization field in the magnet close to the pole edges is high, and it reaches a maximum of $13.9 \mathrm{kOe}$ at the magnet corners that are close to the gap. The minimum intrinsic coercivity, $\mathrm{H}_{\mathrm{cJ}}$, of the $\mathrm{N} 42 \mathrm{SH}$-grade magnet at $20^{\circ} \mathrm{C}$ is $21 \mathrm{kOe}$. Therefore, $13.9 \mathrm{kOe}$ is an acceptable demagnetization field for the 17.2-mm-period undulator. 

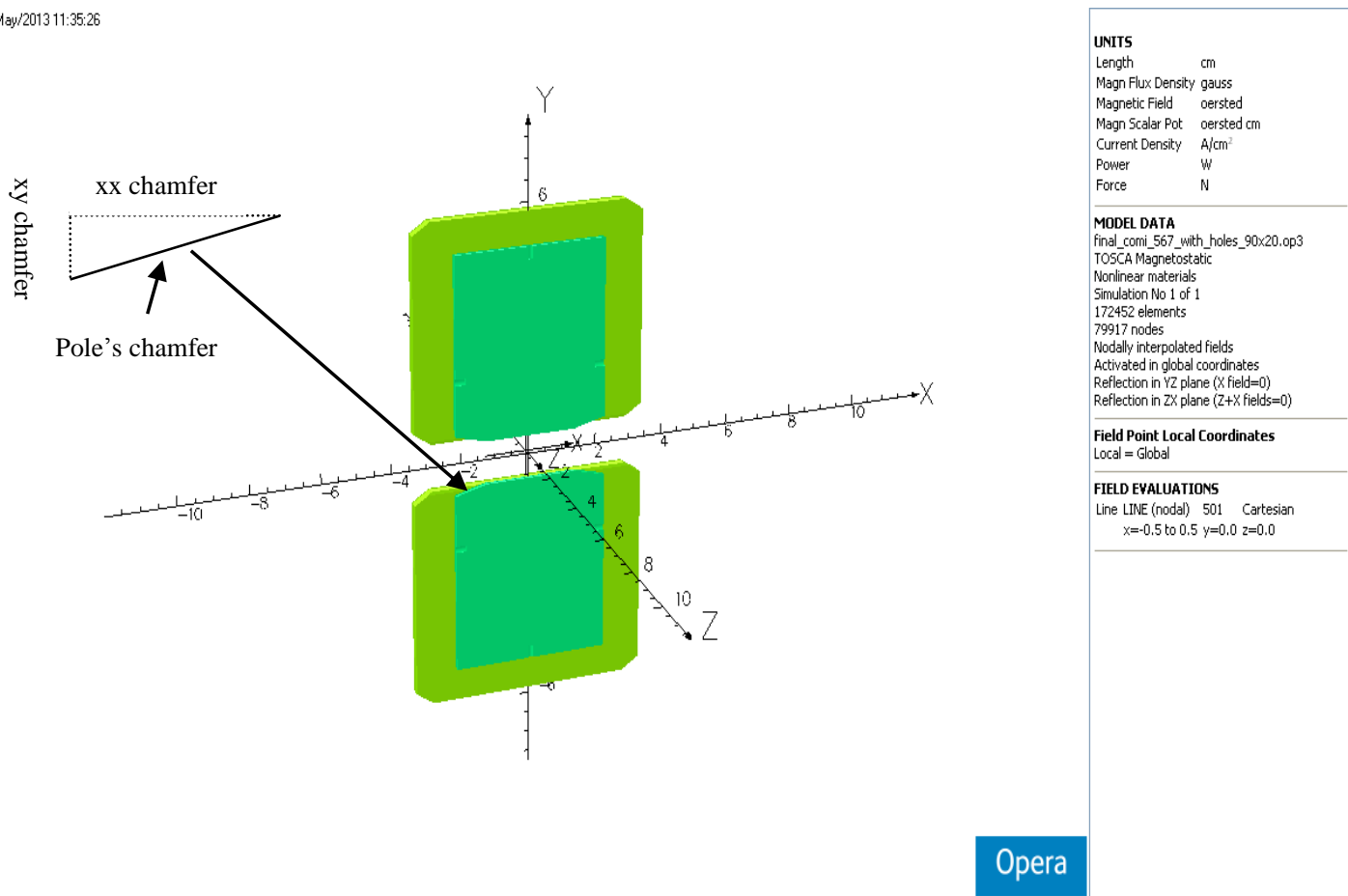

Fig. 1. The quarter-period-long model of a 17.2-mm-period undulator; the light green color is the permanent magnet and the darker green color is the pole in the model.

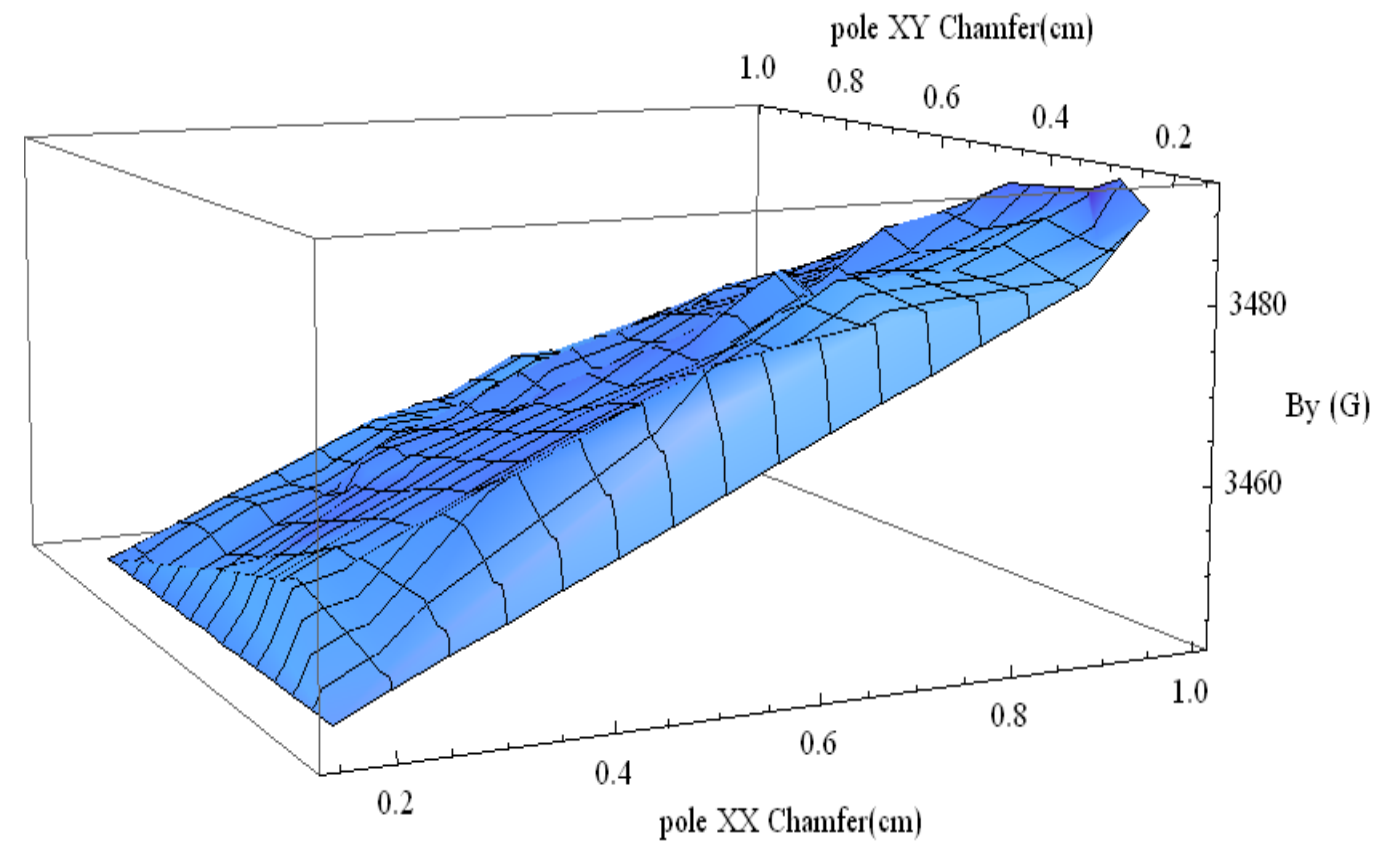

Fig. 2. The calculated peak field (By) as a function of the pole's $x x$ and $x y$ chamfer dimensions for a 17.2mm-period undulator. 


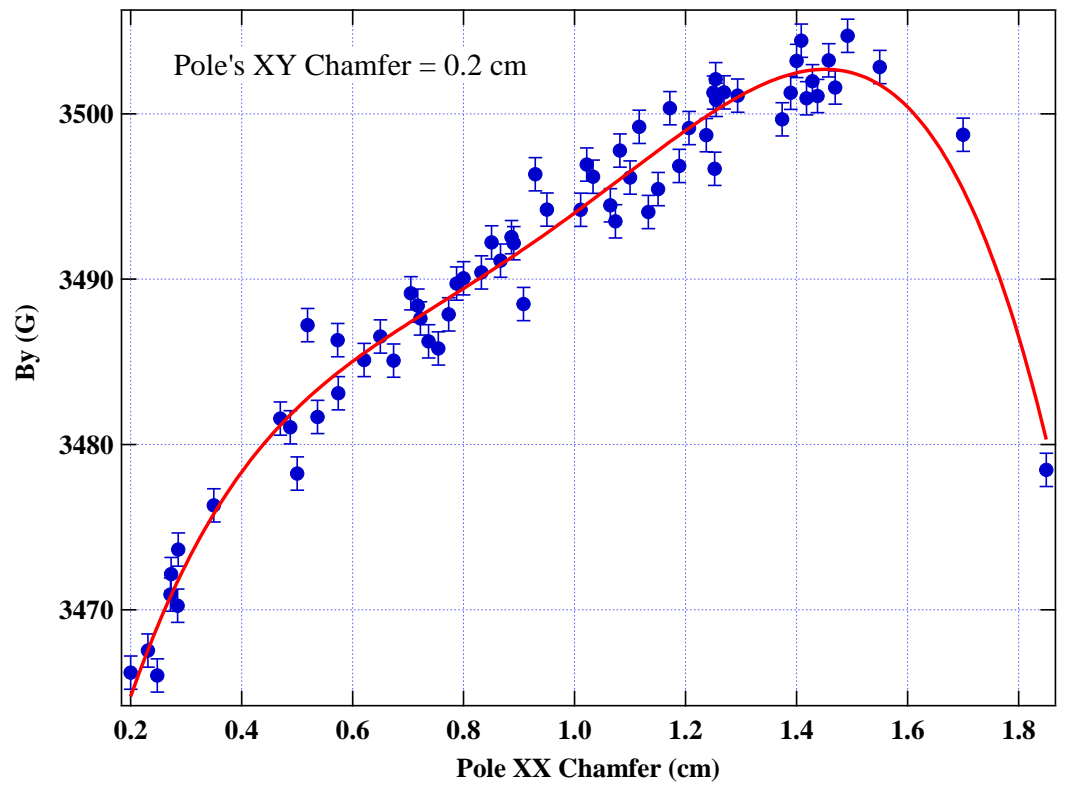

Fig. 3. The calculated peak field as a function of the pole's xx chamfer with the pole's xy chamfer fixed at $0.2 \mathrm{~cm}$ for a $17.2-\mathrm{mm}$-period undulator.

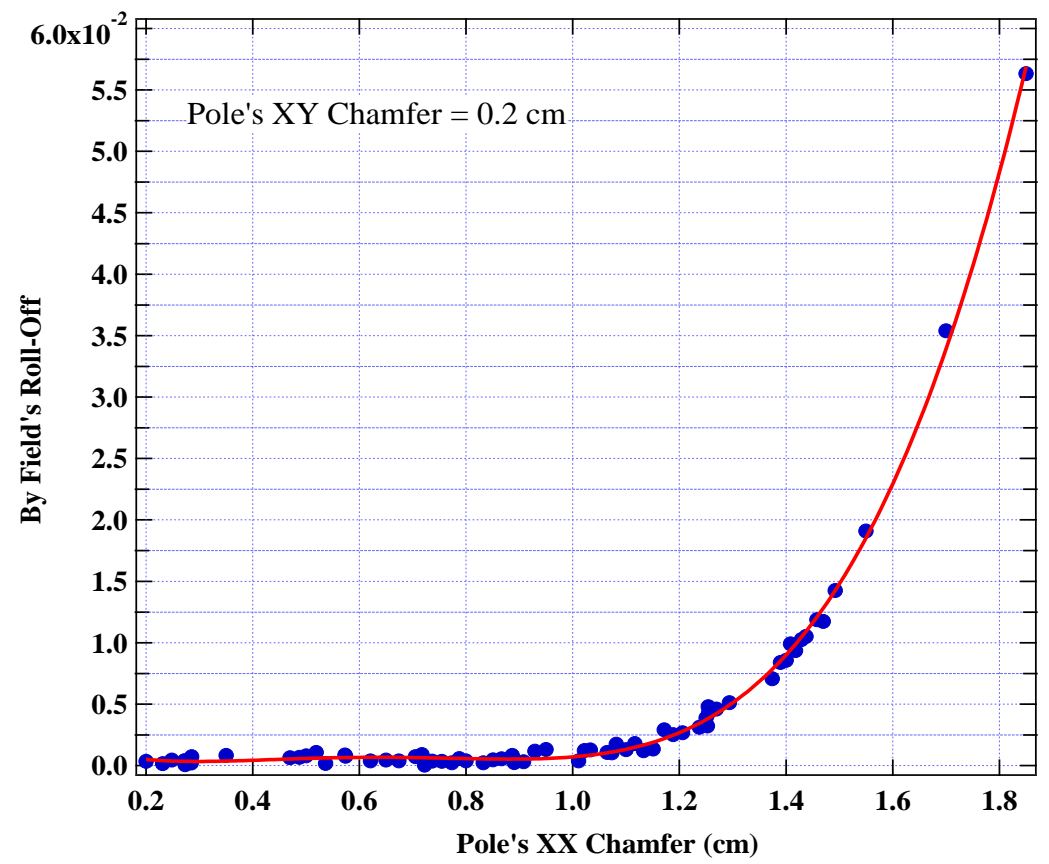

Fig. 4. The field roll-off as a function of the pole's $\mathrm{xx}$ chamfer with $0.2 \mathrm{~cm}$ of the pole's xy chamfer for a $17.2 \mathrm{~mm}$-period undulator. 

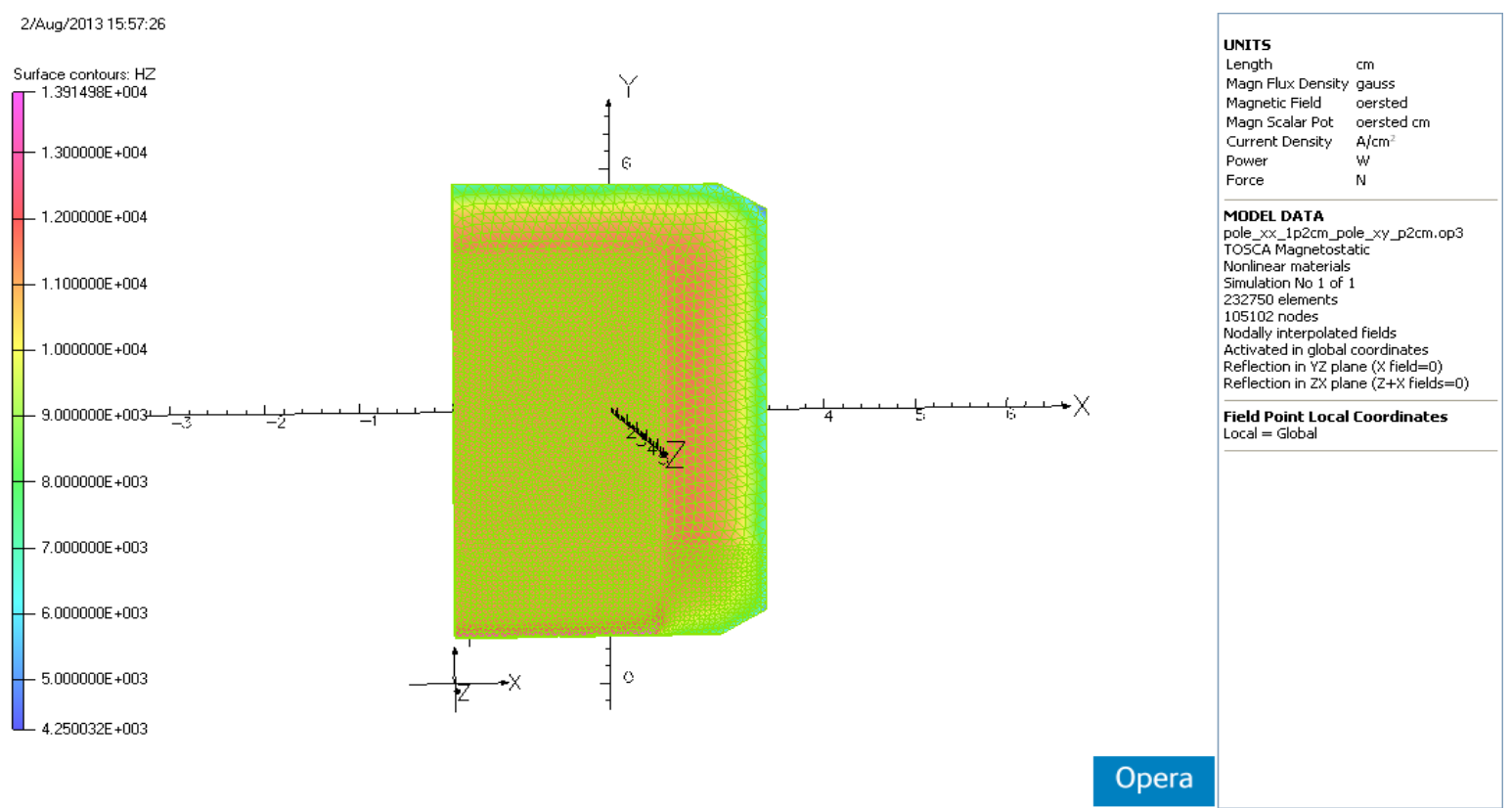

\section{Opera}

Fig. 5. The calculated maximum demagnetization field, $\mathrm{H}_{\mathrm{z}}$, in the magnet block with the pole's $\mathrm{xx}$ and $\mathrm{xy}$ chamfers of $1.2 \mathrm{~cm}$ and $0.2 \mathrm{~cm}$, respectively, at a gap of $10.4 \mathrm{~mm}$ with a $17.2-\mathrm{mm}$-period undulator. Only half of the magnet is shown in this figure.

\section{Conclusion}

The relationship between a hybrid planar undulator's peak field and its roll-off with its pole's xx and xy chamfers has been investigated through the 17.2-mm-period undulator design. Results showed that the proper dimensions for the pole's $x x$ and xy chamfers are $1.2 \mathrm{~cm}$ and $0.2 \mathrm{~cm}$, respectively. The calculated demagnetization field with these chamfers was $13.9 \mathrm{kOe}$, which is small enough compared with maximum demagnetization field, $21 \mathrm{kOe}$, of the permanent magnet for the undulator. 